\title{
Currículo: un campo educativo de polisemia, debates y perspectivas ante los desafíos globales
}

\author{
Curriculum: an educational field of polisemia, debates and \\ perspectives to global challenges
}

\author{
- ROBERT ANTONIO GÓMEZ ESCOBAR \\ escobarroberto2020@gmail.com \\ Código ORCID: 0000-0002-7522-6763 \\ Fundación de Educación para el Emprendimiento, Venezuela
}

Artículo recibido en octubre 2019 / Arbitrado en noviembre 2019 / Publicado en enero 2020

\begin{abstract}
Resumen El proceso de mundialización ha conquistado terrenos económicos, políticos, sociales y culturales por el acelerado crecimiento tecnológico que está experimentando la humanidad. Por lo que se hace necesario reflexionar sobre el papel del currículo y sus entramados teóricos, así como las tensiones en las prácticas educativas ante los desafíos educativos globales presentados por la Unesco, los cuales demandan una educación equitativa, inclusiva y de calidad que brinde un bien común. De allí que esta investigación haya realizado una revisión teórica sobre las posturas conceptuales del currículo según Bobit (1918) y Dewey (1920) y cómo, desde estas, se ha creado un campo de polisemia con influencia en el desarrollo y evaluación de este hasta la actualidad, en la que el currículo es visto como un agente político que se resignifica en los escenarios ideológicos, económicos y culturales, manteniendo una tradición decimonónica de las primeras décadas de siglos XX
\end{abstract}

Palabras clave: Currículo; polisemia; debates teóricos; evaluación curricular; desafíos globales

\begin{abstract}
The globalization process has conquered economic, political, social and cultural lands by the accelerated technological growth that humanity is experiencing Therefore, it is necessary to reflect on the role of the curriculum and its theoretical frameworks, as well as tensions in educational practices in the face of the global educational challenges presented by Unesco, which demand an equitable, inclusive and quality education that provides a good common. Hence, this research has carried out a theoretical review on the conceptual positions of the curriculum according to Bobit (1918) and Dewey (1920) and how, from these, a polysemy field has been created with influence on the development and evaluation of this until the today, in which the curriculum is seen as a political agent that is resignified in ideological, economic and cultural settings, maintaining a nineteenth-century tradition of the first decades of the twentieth centuries.
\end{abstract}

Keywords: Curriculum; polysemy; theoretical debates; curriculum evaluation; global challenges 


\section{INTRODUCCIÓN}

Hoy en día uno de los retos de la ciencia de la educación es lograr el consenso académico en la construcción de una definición de currículo con amplio espectro y alcance para enfrentar los desafíos actuales en diversos contextos, tiempos y espacios. Sin embargo, por ser el currículo un campo flexible y dinámico, debido a que uno de sus objetos de estudio está vinculado con las acciones sociales y culturales, se le ha venido otorgando un carácter polisémico y de agente político, porque se encarga de materializar el ideal educativo desde los procesos y manifestaciones sociales, culturales, científicas y tecnológicas, por consiguiente, lo convierte en un elemento de control y poder en cuanto a que puede mantener cierta hegemonía del conocimiento, en consecuencia, la forma y estilo de cómo plantearlo puede obedecer a pretensiones políticas, ideológicas y económicas en un momento y paradigma determinado.

Con el avenimiento de la sociedad del conocimiento, producto del acelerado desarrollo de la ciencia y tecnología, las exigencias a la educación han sido cada día más amplias, lo cual ha hecho que su campo de estudio se resignifique de acuerdo con los contextos culturales, políticos y económicos. En efecto, la evaluación viene a cumplir un papel fundamental en el desarrollo de la transformación educativa en las últimas décadas, debido a que esta se convierte en un agente de revisión, reajuste y verificación para los cambios requeridos, lo que permitiría articular e incorporar los nuevos conocimientos en la dinámica educativa, en consecuencia, trae consigo un proceso de innovación permanente desde la disciplina misma de la educación para potenciar el proceso inter y transdisciplinario (como una de las últimas tendencias).

Al plantearse la ciencia de la educación las ideas, visión y misión en un contexto y tiempo determinado, el currículo como agente sociocultural, se resignifica para adaptarse a las nuevas realidades y demandas. Esto hace de la evaluación un proceso complejo y dinámico que va a depender de los diversos debates y perspectivas teóricas del currículo, debido a que representa el elemento que materializa tales ideales. En efecto, la evaluación del currículo se convierte en el agente descriptivo del proceso curricular gestionado y brinda los insumos para su transformación. En consecuencia, se orienta por las posturas teóricas, esta permitirá que se organicen los elementos que arrojarán los resultados para los fines y metas planteados el problema persiste en la formas, estilos y cómo llevar el proceso en momentos donde las demandas se hacen cada vez más exigentes, más allá de una educación para todos es construir las bases para una educación equitativa, inclusiva y de calidad, los cuales generan desafíos y metamorfosis dentro del mundo educativo en perspectiva del bien común.

Las posturas teóricas de la eficiencia han tenido grandes influencias en los estilos de evaluación de los aprendizajes y en las últimas décadas del siglo XX van a ocupar los espacios de las transformaciones curriculares, sin embargo, es a partir de las décadas de los 90 del siglo XX cuando se comienza a considerar la evaluación de procesos con elementos emergentes y participativos, lo 
cual plantea un debate de las dicotomías teóricas de la evaluación del currículo en cuanto a su polisemia y los elementos metodológicos para abordarla. Esta es la razón por la cual, en el presente escrito se pretende describir los elementos teóricos y metodológicos que caracterizan tal polisemia y cómo esta influye en la evaluación curricular desde sus entramados teóricos, a la luz de los desafíos que el mundo global le exige a la educación, generando nuevos sentidos y significados del currículo, por ende, el propósito del presente artículo es generar una reflexión sobre el papel del currículo, a partir de sus tensiones teóricas ante los desafíos educativos globales y la influencia en el proceso de evaluación del currículo.

\section{MÉTODO}

En el presente se aplicó un diseño metodológico bibliográfico de tipo documental con fines de plantear procedimientos lógicos en cuanto al análisis, síntesis, deducción lo que permite fundamental los planteamientos mediante un proceso de abstracción teórica.

\section{DESARROLLO Y DISCUSIÓN}

\section{El currículo como elemento de control político}

El currículo es unos de los agentes de la educación que ha generado grandes debates en todos los espacios educativos, debido a que no ha podido concretar su significado y conceptualización porque es expresión de los complejos procesos sociales y políticos, dado a que las estructuras van orientadas por las políticas de los estados-nación. Lo que quiere decir, que cada sociedad experimenta formas y estilos educativos que copian su forma de pensar y actuar ideada en la educación y materializada en el currículo en momentos históricos determinados.

Como lo plantea Gvirt y Palamidessi (1998) "el significado del currículo depende de la forma de cada país y cada tradición pedagógica organiza las prácticas educativas" (p.7). Por ende, el sentido y significado del currículo se encuentra en la diversidad de intereses que cada sociedad define en un tiempo y espacio determinado, aunado a esto, la influencia de otras disciplinas en su conceptualización como son la sociología, la psicología, la antropología, filosofía, entre otras.

En consecuencia, se puede considerar al currículo como un instrumento de continuidad cultural, debido a que permite transmitir todas las creaciones, hallazgos y desarrollos tecnológicos en un tiempo y contexto terminado (Barreto, 2009). Es un agente sociocultural e histórico, que proyecta las necesidades de formación como también la evolución de los procesos académicos y los requerimientos emergentes producto de los avances científicos. Desde esta perspectiva, el currículo es el canal con el que la escuela materializa una estructura política-ideológica, debido a que este es el documento sistematizado, organizado y procesado que lleva implícito un metamensaje que no se detalla a simple vista, sino que su estructura misma está condicionada para dar respuesta a intereses hegemónicos y de esta manera mantiene el control. Al respecto, López (2001) 
"invita a ser leído como manifestaciones concretas de una posición en las estructuras de poder y control hegemónico en un momento histórico determinado" (p.10).

En tal sentido, el currículo se convierte en un instrumento de dominación, a partir de la construcción de marcos académicos que coadyuven a orientar los procesos pedagógicos y didácticos desde líneas estratégicas ligadas al aparato político en un momento histórico. Esto lo resalta Escudero (1999) al exponer "Además, toda concepción del currículo conlleva a un significado político, que concierne a cuestiones relativas a quien deba tomar la decisión" (p. 27).

En efecto, se puede considerar al currículo como un elemento que procesa y sistematiza tácitamente los intereses políticos y económicos ideados en la educación (Uzcátegui, 2011, p. 6), a través, de constructos de imaginarios colectivos que representan toda la memoria de un pueblo, sobre la base de los procesos culturales visibilizados y en algunos casos son invisibilizados. Esta ortodoxa definición describe el sentido de lo que ha venido siendo el currículo en su práctica, en este reposan los procesos y técnicas para enseñar y aprender de forma preestablecida.

Sin embargo, los requerimientos $y$ demandas educativas a nivel mundial han causado que este se resignifique y amplíe su objeto de estudio, trayendo como consecuencia, procesos de rupturas y continuidades, por los cambios económicos y sociales ecuménicos, relacionados con el desarrollo científico y tecnológico que ha provocado crisis en el seno de sus visiones paradigmáticas para generar transformaciones educativas emergentes y contextualizadas.

\section{El currículo entre debates, contextos y perspectivas teóricas}

Para la comprensión de los procesos y el campo del currículo es importante el análisis desde sus perspectivas teóricas. El currículo como elemento de continuidad cultural y político como se describió anteriormente, está orientado por unos debates teóricos que dirigen todas sus prácticas y campos. Desde la perspectiva histórica, es importante ubicarse en las primeras décadas del siglo XX con el surgimiento del vocablo currículo desde las escuelas teóricas estadounidenses, solo se concebía como simple proceso para la organización de las prácticas educativas, donde los elementos centrales de dicha concepción ubicaban su atención en los planes de estudios, la planificación y la evaluación de los aprendizajes. Al respecto Díaz Barriga (2003) expone:

\begin{abstract}
El ámbito curricular surgió con dos tendencias que a finales del siglo $X X$ mostraron sorprendentes desarrollo. Una vinculada a los procesos educativos, las experiencias escolares y el desarrollo de cada estudiante (...) por otra parte, una visión más cercana a las instituciones, es decir a las necesidades de establecer con claridad una secuencia de contenidos que fundamenten la elección de los temas de enseñanzas. (p. 05)
\end{abstract}

Estas tendencias van a marcar la pauta de lo que el autor denomina el campo del currículo, estas son el reflejo de posiciones ideológicas expresadas en los ámbitos educativos, una de ellas nace con el movimiento educativo progresista de John 
Dewey en 1902 desde la obra The child and the currículum, donde propone una educación centrada en los intereses de los estudiantes y la vida cotidiana, y la otra con la representación de Franklin Bobit en 1918, con la obra The Curruculum, centrada en el currículo como proyecto institucional. Durante todo el siglo XX van a tomar fuerzas. Especialmente en los contextos bélicos en los que las ideologías las toman como referencias en el trabajo curricular para mantener sus dominios, expresado en el movimiento de la educación por objetivos, la eficiencia y con ello, la preinscripciones de procesos pedagógicos. En oposición surge la escuela activa, renovadora cuyo centro de atención está en las necesidades del individuo.

Desde las décadas de los 40 hasta mediado de los 90 en América Latina estas tendencias conceptuales del currículo van a tener repercusiones en los procesos curriculares, ejemplo de ello lo tenemos en Venezuela con la llegada de la filosofía de la escuela nueva con el maestro Luis Beltrán Prieto Figueroa, quien promueve la educación desde la filosofía humanista, específicamente con el vínculo establecido con Uruguay y el maestro Sabas Olaizola, quien promueve el método de la escuela activa, materializado en el modelo de escuela experimental Venezuela y Artiga. Esto amparado por una filosofía política y de Estado, representado en un proyecto educativo respaldado en el seno de las instituciones del Estado del momento desde la promulgación de la Ley Orgánica de Educación de 1948, tiempo de contradicciones educativas con las llamadas educación de masas y educación de casta. Posteriormente, el panorama político orienta el campo del currículo hacia la escuela de la eficiencia y el currículo por objetivos dirigido por José Manuel Siso Martínez en las décadas de los 60.

Todas las acciones educativas tuvieron su movimiento político, amparadas en las siguientes obras: Ralph W. Tayler.(1949). Basic principles of curruculum ahd instruction; Benjamín S. Bloom. (1956). Taxonomy of educacional objetiv; B.F. Skinner (1958). Programmed instruction; Taba, Hilda. (1962). Curriculum development theory and practice; Gagné, Robert. (1965). The conditions of lerning; Brumer, Jerome (1966). Toward a theory instruction. Ausubel, David P. (1968). Educational Psychology a cognitive view.

Estas obras son fuentes de primera mano para la comprensión de todo el campo del currículo que se orienta por la visión de la eficiencia y por objetivos, que en Venezuela va a tener grandes influencias en los procesos de transformación curricular, especialmente en las décadas de los 80 del siglo pasado. Sin perder de vista el replanteamiento de la educación desde la visión humanista de Pablo Freire quien entra en contradicciones políticas e ideológicas con las presentadas anteriormente (desde las décadas de los 60).

Es importante destacar que estas posturas teóricas presentadas anteriormente están descritas desde una visión de las décadas de los 80 y 90 del siglo pasado, por lo cual, es importante complementarlas con la visión teórica de la polisemia del currículo que presentan Cano, Portela y Taborda (2018), estos parten que "hay que reiterar que el currículo tiene un espacio, un tiempo y un paradigma" (p. 2019). En su trabajo nos presentan a la polisemia curricular desde las tradiciones teóricas que orientan el campo del 
currículo, entre estas: tradición académica, tradición interpretativa y tradición socio-crítica.

En relación con la tradición académica, esta abarca todo el marco referencial explicado anteriormente, especialmente, la eficiencia. Según los autores las características principales de esta tradición se centran en el currículo como plan de estudio, acumulación de contenidos preinscritos en secuencias con metodología de enseñanza y aprendizaje preestablecidos. En efecto, la actitud pasiva del estudiante es una característica de este campo o tradición curricular. Al punto de hacer del documento curricular una receta de saberes con posibles comportamientos y actitudes que se deben manifestar para lograr los objetivos, todo lo que esté fuera de estos parámetros no es aceptado.

Esta tradición se puede ver reflejada en los modelos y estilos curriculares, pedagógicos y didácticos de la tradición curricular en Venezuela, que prevalecen en los momentos actuales, donde los estudiantes tienen que cumplir con una serie de contenidos pautados y estrategias cumplidas por el docente, este se preocupa más por cumplir los objetivos y contenidos que por verificar el proceso de aprendizaje de los educandos, lo que ha traído como consecuencia que el proyecto curricular en estos momentos de avances tecnológicos no corresponda con la realidad de los aprendices ni de los mismos docentes, es decir, se plantea una realidad ajena donde el individuo para transformarse tiene que cumplir con parámetros al igual que una receta de cocina.

Es en este escenario el debate que, en materia curricular, existe en las instituciones educativas de todos los niveles y modalidades del sistema educativo venezolano, la persistencia de la visión decimonónica de la tradición académica, de la eficiencia y por objetivos ante la necesidad de cambios y transformaciones educativas como un proceso ecuménico. Desde el punto de vista de control político fueron propuestos desde las décadas de los 60 del pasado siglo por los partidos tradicionales hasta mediado de las décadas de los 90 con el documento de educación para todo de la Unesco y posteriormente los pilares de la educación en 1996 con el informe de Delors.

La tradición o paradigma interpretativo propone el diálogo efectivo, crítico, entre lo cognitivo y sociocultural del campo curricular, lo que quiere decir que la escuela no se reduce a un claustro, sino que toma en cuenta las comunidades y otros actores como parte del hecho educativo. Considera como importante los procesos de aprendizaje y lo que en la concepción academicista es considerado extracurricular, en esta tradición es parte del proceso de aprendizaje, es decir, existe un diálogo de los aprendizajes como proceso social. De aquí surge el currículo contextualizado y la relación directa con lo comunitario, orientado por la formación de las humanidades. Todos los elementos familiares, comunitarios, estadales y del interés de los educando son de suma importancia para asumir un diálogo entre saber y hacer para la convivencia ciudadana.

Este paradigma o tradición como se expuso anteriormente fue una propuesta ecuménica para lograr mayor inclusión y calidad educativa a partir de las décadas de los 90 del siglo pasado por la Unesco, este trabajo ha sido de vanguardia y se puede 
evidenciar en los diversos informes de seguimientos de la educación para todos. En especial, al resignificado de la educación y con ello del currículo como agente de inclusión, transformación y participación. En Venezuela, se dieron sus primeros pasos a finales de las décadas de los 90 con el trabajo desde la metodología de proyectos de aprendizajes, donde se llega hasta la educación primaria, media general y técnicas, esto ha sido un punto álgido y de grandes contradicciones, al igual el universitario.

En esta tradición, el hombre es un ser emocional, sujeto político, creador y mucho más que simples contenidos preinscriptivos. Estas posturas humanistas han traído contradicciones en Venezuela, ejemplo de ello se tiene con los procesos de transformación de la educación básica, especialmente en el nivel educativo de media general y técnica. Con la propuesta del currículo bolivariano, en su primer intento en la primera década del siglo $X X I$, el choque paradigmático en el gremio docente causó el fracaso de su discusión e implementación. Aparte de los problemas metodológicos en materia curricular y pedagógica contenidos en el documento, causó la contradicción, a partir, del cambio de visión educativa y con esto, las formas metodológicas para abordar los procesos de enseñanza y aprendizaje, se puede decir, que generó un choque paradigmático en el campo del currículo ante los requerimientos del mundo.

La crisis paradigmática trajo como consecuencia la incomprensión de una polisemia curricular en debate por parte de los entes internacionales y el ente rector del sistema educativo en Venezuela. La incomprensión fue generada por el control y regulación del Ministerio de Educación y la postura de los docentes quienes no aceptaron la propuesta por considerarla política y un mecanismo de control e injerencia política. Aislado de todos los requerimientos globales y la necesidad de una transformación educativa ajustada a las nuevas exigencias mundiales, fue concebida como un aparato político del Estado. Aparte que no se admitía desde el cuerpo docente un cambio curricular que sustituyera los objetivos, contenidos y nuevas formas de planificación de los aprendizajes.

La tradición socio-crítica establece el diálogo etnológico, las culturas, las diversidades, considera al currículo como elemento socio-cultural, cuyo objetivo principal es política, liberadora y emancipadora desde las diversas realidades emergentes poniendo en el centro de las acciones educativas al individuo. Desde esta perspectiva, el hombre es un ser político, de dificultades propias, por ende, el currículo debe ser flexible, abierto y enfocado en las necesidades del ser. Se aprende desde la comprensión e intereses de los educandos. Esto demanda de una madurez educativa de todos los actores en asumir a la educación como un elemento liberador y emancipador del educando donde la postura del docente debe generar la comprensión de los espacios educativos. En esta tradición es fundamental la investigación acción, participativa, liberadora y transformadora. El estudiante es un reto investigativo para el docente donde la dinámica educativa sea contextualizada y un objeto de investigación.

Otra tradición es el currículo oculto, importante para comprender los procesos no 
establecidos dentro del documento curricular, ya que esto viene a representar los momentos y circunstancias que son relativas y efímeras en la puesta en práctica de un proyecto curricular, es lo que no está escrito pero que se pone en relieve durante el desarrollo curricular. Esto no está orientado desde la oficialidad sino de lo emergentes y son estas experiencias las más significativas, donde los actores educativos deben adquirir las habilidades para sistematizarlas y sacarlas a relieve como un proceso significativo. Este es considerado también por Díaz Barriga (2003) como la expectativa de la experiencia, articulada con una serie de aprendizajes no explícitos en el plan de estudio (p. 7).

\section{Contexto de la evaluación curricular}

La evaluación curricular viene a representar a esos contextos de polisemia curricular que se generaron a mediado del siglo $\mathrm{XX}$, específicamente con la tradición teórica de la eficiencia que tiene sus postulados en las base de Bobit en 1918, luego serán replanteadas por Tayler en 1949 y Lewy en 1976. Lo que vino a centrar su atención, en principio, a la evaluación de los aprendizajes centrada en los objetivos y contenidos de las disciplinas, posteriormente en la década de los 70 , se generan los debates en torno al objeto de estudio centrado en el plan de estudio. Se concibe al proceso de evaluación como un medio técnico, fisicalista donde los resultados son interpretados desde una visión estadística y muy numérica.

Es importante destacar, que la visión de la eficiencia del currículo como plan de estudio, centrado en objetivos y contenidos ha tenido sus influencias hasta hoy en día, en todos los sistemas educativos desde básica hasta la educación superior. Es el tema que más ha generado disyuntivas en los procesos de transformación curricular en Venezuela y América Latina, especialmente en la década de los 90 cuando en América Latina, especialmente México y Chile se toman los modelos de evaluación y acreditación de programas educativos de otros países (Díaz Barriga, 2005a, p. 2). Lo que entra en tensión con la evaluación curricular, debido a que esta se considera como una parte minúscula del proceso de evaluación para la acreditación de programas con fines de asegurar y mejorar la calidad educativa, concepción que se viene afianzar en las primeras décadas del siglo XXI.

La evaluación curricular a parte de sus tensiones internas sobre el campo y objeto de estudio orientado por la visión teórica del currículo, se encuentra desplazada o minimizada por la acreditación de programas educativos (Vargas, 2010, p. 7), esta es una práctica que desde el siglo XIX se viene desarrollando en los Estados Unidos de América con fines de mejora de calidad de los programas educativos. En toda América Latina hoy en día es uno de los retos de la educación superior, la evaluación y acreditación que tiene que ver más con los procesos de gestión institucional de forma general pero su existencia es considerada como un mecanismo más financiero que de retroalimentación por el número de instituciones acreditadoras, la demanda de financiamientos y los criterios e indicadores tomados en consideración. Según, Díaz 
Barriga (2005b), señala: "La evaluación se convirtió en la llave que permitía el acceso a una nueva bolsa económica" (p. 1).

Pero la intención de este escrito está enfocada en describir los elementos de la evaluación curricular de acuerdo con sus tensiones teóricas. Lo que se manifiesta en las estructuras y estrategias metodológicas de la evaluación del currículo, a partir de sus influencias teóricas.

\section{Tensiones teóricas de la evaluación curricular}

Una de las tensiones de la evaluación del currículo está en su confusa y diversa concepción desde el currículo, lo que va a influir en la construcción de estrategias para abordar todo el proceso evaluativo (Vargas, 2010 , p. 5). Sin embargo, una de las nociones más significativas que persisten aún es la reduccionista que va orientada hacia el plan de estudio, en este orden de ideas, Brovell (2001) expone "poniendo en cuestión las concepciones tradicionales ligadas al enfoque instrumental y técnico, que fuera hegemónico por mucho tiempo" (p. 102).

Actualmente este ha sido un campo poco estudiado y su estructura teórica de la evaluación van orientadas por el estudio del plan curricular, como una actividad técnica, dejando a un lado la evaluación como proceso, en consecuencia, ha surgido la evaluación como proceso de investigación en el campo para poner a relieve procesos vistos en el desarrollo del currículo, lo que se denomina investigación acción colaborativa. (Brovell, 2001, p. 105)
En esta perspectiva es conveniente exponer las tensiones teóricas presentadas por Díaz (1995). Este autor, mediante un análisis determina tres procesos teóricos que pueden orientar los elementos metodológicos desde sus debates y perspectivas teóricas. Estos son:

\section{Evaluación de todo el plan de estudio o solo un aspecto del plan de estudio}

En este aspecto se presenta la dicotomía entre los autores que consideran que una evaluación debe tomar en cuenta todos los elementos del plan de estudio, para abarcar toda la estructura y verificar su comportamiento global, en contra de esta visión están los autores que parten en tomar solamente en consideración un aspecto del plan de estudio, esto por la complejidad de la evaluación de cada uno de los elementos que lo integran.

Independientemente de la amplitud o los elementos que deben abarcar la evaluación, esta posición solo está enfocada en el currículo como plan de estudio, en consecuencia, tienen una visión técnica con criterio e indicadores preestablecidos.

\section{La conceptualización de la evaluación como actividad técnica o como una investigación en el área de las ciencias sociales}

En esta visión el evaluador solo aplica instrumentos con criterios e indicadores estandarizados, su función es de un técnico evaluador. Tiene que dar cuenta de lo que se solicita, es decir, lleva unos objetivos preestablecidos con instrumentos elaborados para verificar cualquier acción o situación. 
Como actividad de investigación está orientada al tratado de un aspecto del currículo más que dar un juicio de valores se pretende evaluar el contexto y la dinámica de dicho elemento a considerar. Esta es una postura que en los momentos actuales está tomando importancia en el campo del paradigma cualitativo de la investigación. En este aspecto cabe la propuesta de Brovell (2001) que la denomina investigación acción colaborativa, la cual viene a fortalecer la evaluación como proceso para tomar en consideración las situaciones que emergen en diversos contextos. También Cevera, Martí y Ríos (2014) expresan "debe ser un acto privilegiado de análisis de los quehaceres, prácticas y vidas cotidianas de la comunidad educativa" (p. 7).

\section{La finalidad de la evaluación: tomar decisiones $y$ ofrecer elementos de comprensibilidad del fenómeno educativo}

Este aspecto se reflexiona sobre el para qué de la evaluación y tiene punto de encuentro ante las dos posturas descritas anteriormente, sobre la evaluación como simple requerimiento de verificar el proceso educativo desde una visión técnica para llevar un expediente o para mantener la calidad de la educación. Ante esto, surge la postura de la evaluación como toma de decisiones, que está vinculada al proceso investigativo pero desde la perspectiva administrativa. En contraposición surge la postura que la evaluación contribuye a tener una visión más rica del hecho educativo y de la realidad en que este se genera, en ese sentido, el investigador opera como un investigador y productor de conocimiento en la comprensión del contexto social.

Estas son tensiones que aún persisten en el campo de la evaluación, por ende, se puede afirmar que la evaluación aún es un campo que está en plena discusión sobre su abordaje teórico en cuanto a su objetivo central que está determinado por los contextos políticos, económicos y de control, ejemplo de ello, lo tenemos con el manejo de los resultados, proceso vital en todo acto evaluativo y desde la visión técnica se mantiene la confidencialidad y no se socializa, en las visiones orientadas hacia el acto investigativo se obtienen resultados pero aún falta la socialización con todos los actores.

Ante esto, la evaluación orientada en la toma de decisiones viene a comprender el hecho educativo desde la socialización de los resultados para fortalecer los procesos, el cual tiene una visión de la evaluación como un hecho de retroalimentación para mejorar y avanzar, al respecto, el evaluador antes de ser un agente punitivo y señalador se convierte en un mediador que sostiene el dialogo para la mejora.

\section{La evaluación curricular: estrategias metodológicas desde sus entramados teóricos}

Uno de los problemas que se presenta en la evaluación curricular, se centra en las estrategias metodológicas para abordar el proceso evaluativo desde los diversos entramados teóricos presentados anteriormente. Se podría considerar sensible el campo metodológico de la evaluación curricular por ser una acción joven que desde 
la década de los 70 del siglo pasado se orientaba únicamente a considerar los aspectos de la evaluación de los aprendizajes. Es en la década de los 80 cuando se comienza abordar la evaluación interna y externa del plan de estudio (Díaz Barriga, 2005) y en los 90 surge el proceso de auto-evaluación de programas desde el proceso de acreditación de proyectos educativos.

Díaz (1993) define a la evaluación interna como aquella que va dirigida a evaluar los procesos internos del plan de estudio y la evaluación externa está dirigida al estudio de egresos, campo profesional, mercado ocupacional y el estudio de los avances científicos y tecnológicos de cada una de las disciplinas que integran el saber. Lo que significa que la evaluación del currículo está caracterizada por lo interno que tiene que ver con la dinámica del plan de estudio y el estudio de los factores que influyen en el rendimiento académico, entre otros. Es importante destacar, que la comprensión de cada uno de los criterios e indicadores que conforman lo interno y externo del currículo es digno de un proceso investigativo, lo que permitiría ampliar el campo de la evaluación de cara a las sugerencias de aspectos $y$ metodologías desde una postura sociocrítica.

En relación con la visiones de la evaluación como un hecho investigativo Díaz Barriga (1995) presenta una estructura a tomar en cuenta, constituida por aspectos que llevan a comprender la dinámica emergente del currículo con una visión más crítica en cuanto a los aspectos pospuestos: fundamento del currículo, estudio sobre el desempeño del egresado, fundamentos del plan de estudio, análisis de las perspectivas del estudiante, estudios de corte etnográfico y la situación docentes y estudiantes.

Con una visión también socio-crítica, Bovell (2001) nos presenta la estructura de una evaluación curricular caracterizada por una evaluación interna como proceso intrínseco que toma en consideración la congruencia interna y compara lo escrito en el proyecto curricular y lo vivido, además que le da importancia a la evaluación de los resultados, importante elemento, porque viene a fundamentar lo expuesto anteriormente sobre el tema, la autora parte de que los resultados deben ser evaluados por todos los actores y la comunidad para llevar a la reflexión y generar acciones en conjunto, lo que requiere de construir una cultura de evaluación colaborativa.

Para esta autora la evaluación externa la constituyen los agentes externos como los egresados, jefes de puestos profesionales, analistas académicos y profesionales en el área. Incorpora el elementos de la rendición de cuentas, a esto se le atribuye un carácter oficial porque va dirigida por los entes oficiales, ministerios y oficinas de educación, entre otras.

Se deja a la reflexión que la metodología curricular es un campo poco explorado y complejo en su comprensión debido a las diversas visiones teóricas y tensiones polisémicas dada desde la visión del currículo.

\section{Una era de civilización y tensiones educativas ecuménicas}

Con el avenimiento de la tecnología en todos los ámbitos de la vida social de la actualidad, la tecnología de la información y comunicación y la presencia de herramientas digitales y artefactos tecnológicos de 
avanzados procesamientos de información, se han generado procesos de rupturas y continuidades culturales, evidenciados en las transformaciones de sistemas de valores y creencias de las sociedades actuales, proyectados y adaptados como moda en los individuos de a pie, cuya dinámica, está orientada por la llamada "aldea global" y sociedad del conocimiento. Estas se han hecho presentes, a través, de códigos y parámetros estándares globales, superando, por así decirlo de forma metafórica, la tabla periódica y la codificación de sus elementos por códigos digitales que pretenden llegar en todos los rincones del mundo con sistemas culturales establecidos, haciendo de la cultura un proceso digital ecuménico.

En este caso, se pudiera decir, que estamos ante un proceso de innovación y transformación partiendo del replanteamiento de nuevas formas de actuar y pensar por el impulso de la ciencia y tecnología, en efecto se evidencia un cambio civilizatorio, propio del sistema evolutivo natural del hombre en sociedad con características de universalización y tecnología, este último desde su entendimiento histórico, como motor de la historia para las transformaciones de las sociedades. Donde los procesos de transformaciones se manifiestan en los elementos culturales proyectados por los individuos como resultado del expansionismo ideológico con nuevas formas de conquista y afianzamiento desde la génesis de un orden mundial. Ante esta postura Aranguren (2013) afirma "Los cambios acelerados, crean fisuras y rupturas en el modelo pedagógico tradicional, de estilo cartesiano, poniendo en tela de juicio a las funciones culturales" (p. 40).
Sin embargo, los trabajos emitidos por la Unesco han generado contradicciones con los intereses económicos y la formación del ser, desde una educación inclusiva, de calidad y para toda la vida. Ejemplo de ello se evidenció en la década de los 90 del siglo pasado con el documento emitido por la Comisión Económica para América Latina y el Caribe (CEPAL): "Educación y Conocimiento: Eje de la transformación Productiva". (1992) y la Conferencia Mundial de la Educación para Todo (Unesco, 1990. Jomtien- Tailandia). Desde este escenario, comienza una dinámica de ajuste educativo, donde el mundo del Internet, la tecnología celular y las computadoras portátiles, exigen un giro pedagógico para adiestramiento y capacitación de "recursos humanos" además de una escuela que permita desarrollar procesos cognitivos efectivos para enfrentar los cambios que la tecnología proyectaba y sigue en proyecciones continuas, como consecuencia surge el consenso de Washington en la década de los 90, y la influencia en todos los procesos de reformas educativas de casi todos los países latinoamericanos. Esto lo confirma Plà (2010), cuando afirma:

La publicación en 1992 de Educación y conocimiento: Eje de la Transformación productiva con equidad, a cargo de la Comisión Económica para América Latina y el Caribe (CEPAL) y de la organización de Naciones Unidas para la Educación, la Ciencia y la Cultura (UNESCO), ordenó el banderazo de salida para llevar a cabo las reformas educativas, con especial 
énfasis en la educación secundaria, que transformaron los sistemas educativos nacionales en América Latina. (p. 66)

\section{La educación y el currículo ante los desafíos globales}

La década de los 90 del siglo pasado se ha convertido en uno de los momentos coyunturales para la comprensión de la educación desde una perspectiva política, ideológica y económica. En ese período el boom de la tecnología saca a relieve los grandes impulsos en tecnología móvil conjuntamente con la aparición del Internet y los artefactos como las laptop (PC) y las computadoras de mesa. Por lo cual, la demanda educativa se hacía cada vez más grande.

Hoy en día la demanda ha crecido y sigue exigiendo cambios con celeridad en los sistemas educativos, por lo cual se plantea una educación orientada al desarrollo del aprendizaje, lo que significa que la educación no es vista como proyecto educacional sino de desarrollo humano. Así lo describe Angulo (2013) "La escuela está llamada a reestructurar la base del conocimiento que se detecta y enseña. Múltiples conocimientos, tareas, actividades, oficios más allá de simple materias" (p. 116).

Por lo tanto, la educación en el siglo XXI se convierte en un proceso complejo donde el centro del quehacer pedagógico está enfocado en el individuo y sus procesos intrínsecos. Por estas razones, la Unesco (2015b) propone replantear la educación para un bien común, reafirmando una visión humanista, "se puede afirmar que mantener y aumentar la dignidad, la capacidad y el bienestar de la persona humana con relación en relación con los demás y la naturaleza" (p. 38).

Es así, como el aprendizaje ante toda la vida se convierte en el principio organizador de la educación, lo que trae consigo nuevos estilos pedagógicos, su implementación está argumentada en la consigna de la educación de calidad, inclusiva y para el desarrollo sostenible desde una visión de justicia social. En consecuencia, se presentan nuevos desafíos educativos en la formación de profesores para la educación de las nuevas generaciones a la par con los requerimientos de las aceleradas dinámicas tecnológicas. Lo que demanda nuevas estructuras epistemológicas de abordar las realidades educativas desde contextos emergentes y transdisciplinarios del ser como protagonista del hecho pedagógico. Ante eso, Amadio, Opertti y Tedesco (2014) expresan "hoy se aboga por una enseñanza que favorezca el aprendizaje activo, centrada en las necesidades y expectativas de los estudiantes considerados como los protagonistas principales" (p. 4).

En consiguiente, la educación según la Unesco (2017) pasa a ser "un aprendizaje que se caracteriza por ser deliberado, intencionado con un fin determinado y organizado" (p. 20). De allí pues, se necesita un currículo con características de globalidad $\mathrm{O}$ integralidad donde principio básico sea la inter para la transdisciplinariedad. Por tal motivo, estas descripciones del contexto educativo y las implicaciones del siglo XXI forman parte de un ideario educativo, así lo afirma Angulo (2013) "el ideal de la educación es tal vez un imaginario colectivo, una concepción de la sociedad, la forma para conseguirlo será el currículo" (p. 106). 
En este orden de ideas, Vargas (2017) la define como

Un proceso a lo largo de la vida que va mas allá de los sistemas educativos, en el que se pone a la persona que aprende, sus necesidades y aspiraciones, en el centro del proceso y en el que se prioriza que los individuos asuman la responsabilidad de su propio aprendizaje a lo largo de sus vidas. (p. 10)

En este sentido, el currículo juega un papel importante, ya que se convierte en el motor básico para articular los desafíos globales educativos y sus tenciones políticas, económicas, culturales y sociohistóricas, razón por la cual, se puede considerar como un agente social que permite la concreción del ideal educativo en realidades emergentes. También es un instrumento de continuidad cultural, debido a que permite transmitir todas las creaciones, hallazgo, descubrimientos y procesos tecnológicos en un tiempo y contexto definido.

\section{CONCLUSIONES}

Uno de los elementos fundamentales que permitiría una transformación curricular, entendida desde el cambio de paradigma, sería la comprensión de la polisemia del currículo en perspectiva histórica, lo que daría una comprensión general a los docentes del cambio curricular desde una necesidad ecuménica más no política partidista. En este sentido, el currículo oculto viene a representar una de las fortalezas para los procesos de transformación curricular, debido a que permitiría poner a relieve situaciones propias de los contextos, para esto es indispensable el desarrollo de habilidades investigativas desde el enfoque cualitativo y en especial la investigación acción participativa. En este caso, la evaluación del currículo es un campo complejo debido a sus tensiones, debates y polisemias como resultado de la amplitud del campo del currículo, en consecuencia, la concepción curricular asumida va a tener influencia en el proceso de evaluación, en especial atención para definir el objeto de evaluación y sus estrategias metodológicas.

La evaluación curricular debe ser un acto investigativo porque permitiría generar un conjunto de proposiciones desde una estructura emergente y con ello poder contextualizarlo en la dinámica política, social, cultural y económica. Sin embargo, es un tema álgido porque de esta forma se descentraliza, cuestión que amenaza a sectores que aún mediante los procesos curriculares mantienen el control del poder.

Es importante activar la investigación en la evaluación curricular para el fortalecimiento de los criterios e indicadores de la evaluación interna y externa desde una perspectiva cualitativa con la incorporación activa de todos los actores del quehacer educativo. En efecto, en los momentos actuales se puede decir que el verdadero sentido y significado de la educación aún está en debate ante los diversos enfoques epistémicos y de intereses políticos e ideológicos que persisten en el mundo. Todo esto es producto del cambio civilizatorio que se está experimentado, trayendo como consecuencia las luchas culturales entre 
Oriente y Occidente, ejemplo de ello se tiene en las complejas transformaciones curriculares en la educación universitaria y el mundo de las competencias, consideradas un mecanismo ideológico de dominación más que por el mercado, sus intenciones son socioculturales.

La sostenibilidad y el enfoque de la educación como un bien común, traen consigo el replanteamiento de nuevas políticas educativas de mayor alcance y de amplio espectro, en consecuencia, generan contradicciones paradigmáticas para entender el hecho educativo y con ello plantear nuevas formas pedagógicas y didácticas en pro al desarrollo de aprendizajes a lo largo de toda la vida. Los procesos de transformación curricular tienen nuevas implicaciones $y$ escenarios que han generado tensiones $y$ desafíos educativos, en especial atención a los procesos inter y transdisciplinarios bajo una concepción humanista de la educación, lo que genera una crisis paradigmática a la hora de dar abordaje pedagógico y didáctico, aun cuando persista el paradigma tradicional del currículo preinscrito. En este contexto hay que dar respuesta al aprendizaje a lo largo de toda la vida, es un principio educativo que debe tener mayor tratamiento epistémico en las formas de abordaje pedagógico y didáctico.

\section{REFERENCIAS}

Amadio, M., Opertti, R. y Tedesco, C. (2014). Un Currículo para el Siglo XXI: Desafíos, Tensiones y Cuestiones Abiertas. En Investigación y Prospectiva en Educación. Documentos de Trabajo. Ginebra-Suiza:
Unesco- Oficina Internacional de Educación (OIE)

Angulo, L. (2013). El Currículo como proyecto de desarrollo humano desde una perspectiva compleja. Teoría y Didáctica de las Ciencias Sociales. Mérida-Venezuela, enero-diciembre. 19, 103-119

Aranguren, C. (2013). Enseñar Ciencias Sociales en un mundo de complejidades $e$ incertidumbres. Reflexiones y Propuestas. Teoría y Didáctica de las Ciencias Sociales. Mérida- Venezuela. 19, 37-47. Recuperado de

http://www.redalyc.org/pdf/652/65232225 003.pdf

Barreto, N. (2009). Seis Momento del Currículo durante el siglo XX y el primer lustro del siglo XXI. UPEL-IPM-MSM: Caracas Venezuela. Central de Venezuela-Facultad de Humanidades y Educación. CaracasVenezuela

Bovell, M. (2001). Evaluación Curricular. Fundamentos de Humanidades Universidad Nacional de San Luis. Año 11n०2

Cano, S., Portela, H. y Taborda, J. (2018). La polisemia del currículo: Influencias, tradiciones y supuestos. Latinoamericana de estudios educativos. Universidad de Caldas. Colombia. 14 (2), 2013-237

Cevera, D., Marti, M., Ríos, D. (2014). Evaluación y acreditación de la evaluación superior: Tendencias, prácticas y pendientes en torno a la calidad educativa. Atenas, 3(27)

CEPAL y Unesco. (1992). Educación y Conocimiento: Eje de la Transformación Productiva con Equidad. Oficina Regional de Educación para América Latina y el Caribe Unesco y CEPAL. Santiago de ChileChile

Delors, J. (1996). La Educación encierra un Tesoro. Informe a la Unesco de la comisión internacional sobre educación del siglo XXI. Ediciones de la Unesco 
Díaz, A. (1995). La evaluación curricular. Un acercamiento a los elementos centrales de esta disciplinan y su ubicación en México. Cuadernos pedagógicos universitarios. $\mathrm{N}^{\circ}$ 8.Colina: Universidad Autónoma de Colinas

Díaz Barriga, A. (2003). Curricular. Tensiones conceptuales prácticas. Revista electrónica de investigación educativa, 5(2)

Díaz Barriga, A. (2005a). Evaluación curricular y evaluación de programas con fines de acreditación. Cercanías y desencuentros. Conferencias para el congreso nacional de investigación educativa

Díaz Barriga, A. (2005b). Riesgo de lo sistemas de evaluación y acreditación de la educación superior. Documento preparado para "seminario regional: Las nuevas tendencias de la evaluación y acreditación en América Latina y el Caribe" Organizado por la CONAE y el IESAL-Unesco, Buenos Aires

Díaz, F. (1993). Diseño curricular II. ILCE, México

Escudero, J. (1999). Diseño desarrollo e innovación del currículo. Madrid: Editorial Síntesis

Gvirtz, S. y Palamidessi, M. (1998). El ABC de la tarea docente: Currículo y enseñanza. Buenos Aires: Editorial Aique

Hutton, W y Giddens, A. (2001). En el Límite: La vida en el capitalismo global. Barcelona: Editores Tusquets

López, N. (2001). La desconstrucción curricular. Colección seminario. Bogotá

Luna S., C. (2015). El Futuro del Aprendizaje 3. ¿Qué tipo de Pedagogía se necesita en el siglo XXI? Educación. Investigación y Prospectiva en Educación. Unesco
Opertti, R. (2011). Visión del Currículo y Debates Curriculares: Una Perspectiva Interregional. En Investigación y Prospectiva en Educación Documentos de Trabajo. Ginebra-Suiza: Unesco- Oficina Internacional de Educación (OIE)

Plà, S. (2010). Las Competencias: Eje para la Transformación del Conocimiento Histórico Escolar. Un Estudio Comparativo en América Latina. Universidad Nacional Autónoma de México. Ciudad de MéxicoMéxico

Unesco. (2015). Leyes generales de educación en América Latina. El derecho como Proyecto Político. Buenos Aires: Unesco

Unesco. (2015b). Replantear la Educación. ¿Hacia un Bien Común Mundia? Educación. Unesco. Investigación y Prospectiva en Educación

Uzcátegui, R. (2011). Elementos para la conformación de una historia del Currículo desde la memoria educativa venezolana. UCV. Facultad de Educación y Humanidades. Escuela de Educación. Caracas-Venezuela

Vargas, M. (2010). La evaluación curricular y la acreditación de programas educativa, desplazamiento y desarrollo. Documento preparado para el $\mathrm{X}$ congreso nacional de investigación educativa. Área 2 currículo

Vargas, C. (2017). El Aprendizaje a lo largo de toda la vida desde una perspectiva de justicia social. En Investigación y Prospectiva en Educación Documentos de Trabajo. Ginebra-Suiza: Unesco- Oficina Internacional de Educación (OIE)

Wallerstein, I. (1998). Después del liberalismo. Madrid: Ediciones: Siglo Veintiuno 